\title{
Robotic Nipple-sparing Mastectomy and Immediate Breast Reconstruction with Gel Implant
}

\author{
Hung-Wen Lai, MD, PhD*†+\$II\#\# \\ Shih-Lung Lin, MD** \\ Shou-Tung Chen, MD*++ \\ Shu-Ling Chen, MS*+ \\ Ya-Ling Lin, BS* \\ Dar-Ren Chen, MD*++ \\ Shou-Jen Kuo, MD ++
}

Background: The experience of application of robotic surgery platform in the management of breast cancer was limited. The preliminary experience and results of robotic nipple-sparing mastectomy (R-NSM) and immediate breast reconstruction (IBR) with Gel implant was reported.

Methods: The medical records of patients who underwent R-NSM and IBR with Gel implant for breast cancer during the period March 2017 to October 2017 were collected from single institution. Data on clinicopathologic characteristics, type of surgery, method of breast reconstruction, complications, and recurrence were analyzed to determine the effectiveness and oncologic safety of R-NSM. Patients' oriented cosmetic outcome report was also obtained.

Results: A total of 15 patients was analyzed, and the mean age of them was $46.5 \pm 10.0$ years. The pathologic stage was $30.8 \%$ ductal carcinoma in situ, $30.8 \%$ stage I, $30.8 \%$ stage II, and $7.7 \%$ stage III. The mean operation time was $282.8 \pm 70.4$ minutes, and mean hospital stay was $6.7 \pm 1.2$ days. The positive surgical margin rate was $0 \%$. One patient suffered from delayed axillary wound healing. Two patients $(13.3 \%)$ with transit nipple ischemia change, but no total nipple areolar complex necrosis case was observed. No local recurrence, distant metastasis, or case mortality was found during mean $6.3 \pm 2.1$ months follow-up. All 15 patients were satisfied with the postoperative aesthetic outcome.

Conclusion: From our preliminary experience, R-NSM and IBR with Gel implant is a safe procedure, with good cosmetic results, and could be a promising new technique for breast cancer patients indicated for mastectomy. (Plast Reconstr Surg Glob Open 2018;6:e1828; doi: 10.1097/GOX.0000000000001828; Published online 11 June 2018.)

\section{INTRODUCTION}

Robotic surgery, which incorporated 3-dimensional imaging system and flexibility of robotic arm and instruments, had been growingly used in different field of surgeries. ${ }^{1-4}$ Robotic nipple-sparing mastectomy (R-NSM), which introduce da Vinci surgical platform through a

From the *Endoscopy E Oncoplastic Breast Surgery Center, Changhua Christian Hospital, Changhua, Taiwan; †Division of General Surgery, Department of Surgery, Changhua Christian Hospital, Changhua, Taiwan; $\neq$ Comprehensive Breast Cancer Center, Changhua Christian Hospital, Changhua, Taiwan; \$School of Medicine, National Yang Ming University, Taipei, Taiwan; IDivision of Breast Surgery, Department of Surgery, Yuanlin Christian Hospital, Yuanlin, Taiwan; ||Minimal Invasive Surgery Research Center, Changhua Christian Hospital, Changhua, Taiwan; **Division of Plastic and Reconstructive Surgery, Department of Surgery, Changhua Christian Hospital, Changhua, Taiwan ; and \# Kaohsiung Medical University, Kaohsiung, Taiwan.

Copyright @ 2018 The Authors. Published by Wolters Kluwer Health, Inc. on behalf of The American Society of Plastic Surgeons. This is an open-access article distributed under the terms of the Creative Commons Attribution-Non Commercial-No Derivatives License 4.0 (CCBY-NC-ND), where it is permissible to download and share the work provided it is properly cited. The work cannot be changed in any way or used commercially without permission from the journal. DOI: $10.1097 / \mathrm{GOX} .0000000000001828$ small axillary wound to perform NSM with (or without) immediate breast reconstruction (IBR), was reported to have potential to overcome the technique difficulty of endoscopic-assisted NSM and showed promising cosmetic outcome. ${ }^{5,6}$ In this study, we report the technique, and

Received for publication February 20, 2018; accepted April 18, 2018.

Supported by the Ministry of Science and Technology of Taiwan, and the number of this funding was: 104-2314-B-371-006-MY3. This study was also sponsored by research funding provided by the Changhua Christian Hospital 104-CCH-ICO-006, and 106-CCH-IRP-011.

Drs. Shih-Lung Lin and Hung-Wen Lai contributed equally to this work. Synopsis: Robotic nipple-sparing mastectomy and immediate breast reconstruction with Gel implant performed through small axillary incision is a safe procedure, with good cosmetic results, and could be a promising new technique for breast cancer patients indicated for mastectomy.

Disclosure: The authors have no financial interest to declare in relation to the content of this article. The Article Processing Charge was paid for by the authors.

Supplemental digital content is available for this article. Clickable URL citations appear in the text. 
preliminary experience and clinical outcome of R-NSM and IBR with Gel implant.

\section{R-NSM TECHNIQUE}

After preoperative marking, the patient was placed in the supine position with operative arm abducted about 90 degrees. The operating side shoulder was elevated to 30 degree with draping (or the arm in operating field was adducted after axillary surgery) to prevent conflict between the operating table and docking of robotic surgery system (Fig. 1A). A physiological saline solution containing lidocaine $0.05 \%$ and epinephrine $1: 1,000,000$ was injected subcutaneously into the whole breast to minimize bleeding. Then an approximately $2.5-4 \mathrm{~cm}$ oblique axillary incision (depend on the size of the breast to be removed) was made over the extramammary region, and axillary lymph node surgery was performed if indicated.

\section{ROBOTIC DOCKING AND DISSEGTION}

To create the working space for the placement of the single port (Glove Port, Nelis, Gyeonggi-do, Korea, Fig. 1B), the subcutaneous flap was dissected with electrocautery under direct vision in a $2.5-4 \mathrm{~cm}$ area. After port placement, carbon dioxide $\left(\mathrm{CO}_{2}\right)$ was inflated with air pressure kept at $8 \mathrm{~mm} \mathrm{Hg}$ to create space for mastectomy. ${ }^{5,7}$ Then robotic side cart (da Vinci, Intuitive Surgical, Sunnyvale, Calif.) is positioned posterior to the patient with the 2 robotic arms and the endoscope extending over the patient in proximity to the ports. In this position, the arms are aligned with the plane of the breast, nearly parallel to the floor (Fig. 1C), and the ports are docked to the robotic arms.

Then the operation was shifted to da Vinci Si (Intuitive Surgical, Sunnyvale, Calif.) robotic platform with operating surgeon controlled at the console. To prevent conflicts during dissection, the elbows of robotic arms were opened as much as possible. We use a 30-degree 12-mm-diameter camera (Intuitive Surgical, Denzlingen, Germany) in the upper port to prevent collisions of other instruments. Dissection was carried out with an $8 \mathrm{~mm}$ monopolar scissor (Intuitive Surgical, Sunnyvale, Calif.). Traction and counter-traction, along with maintaining exposure and stretching out the tissue was carried out with $8 \mathrm{~mm}$ prograsp forceps (Intuitive Surgical, Sunnyvale, Calif., Fig. 1B). The location of scissor and prograsp in right or left arm could be adjusted during operation if needed.

The dissection started from the superficial skin flaps in all quadrants. To facilitate skin flap dissection, tunneling technique was applied, ${ }^{8-10}$ and the septa between the skin flap and parenchyma were dissected using monopolar scissors. Then subnipple biopsy was performed by taking 2 separate specimens (inner and outer part) under the nipple areolar complex (NAC) and the intraoperative frozen section was analyzed. ${ }^{11}$ After completion of superficial skin flap dissection, the peripheral and posterior dissec-
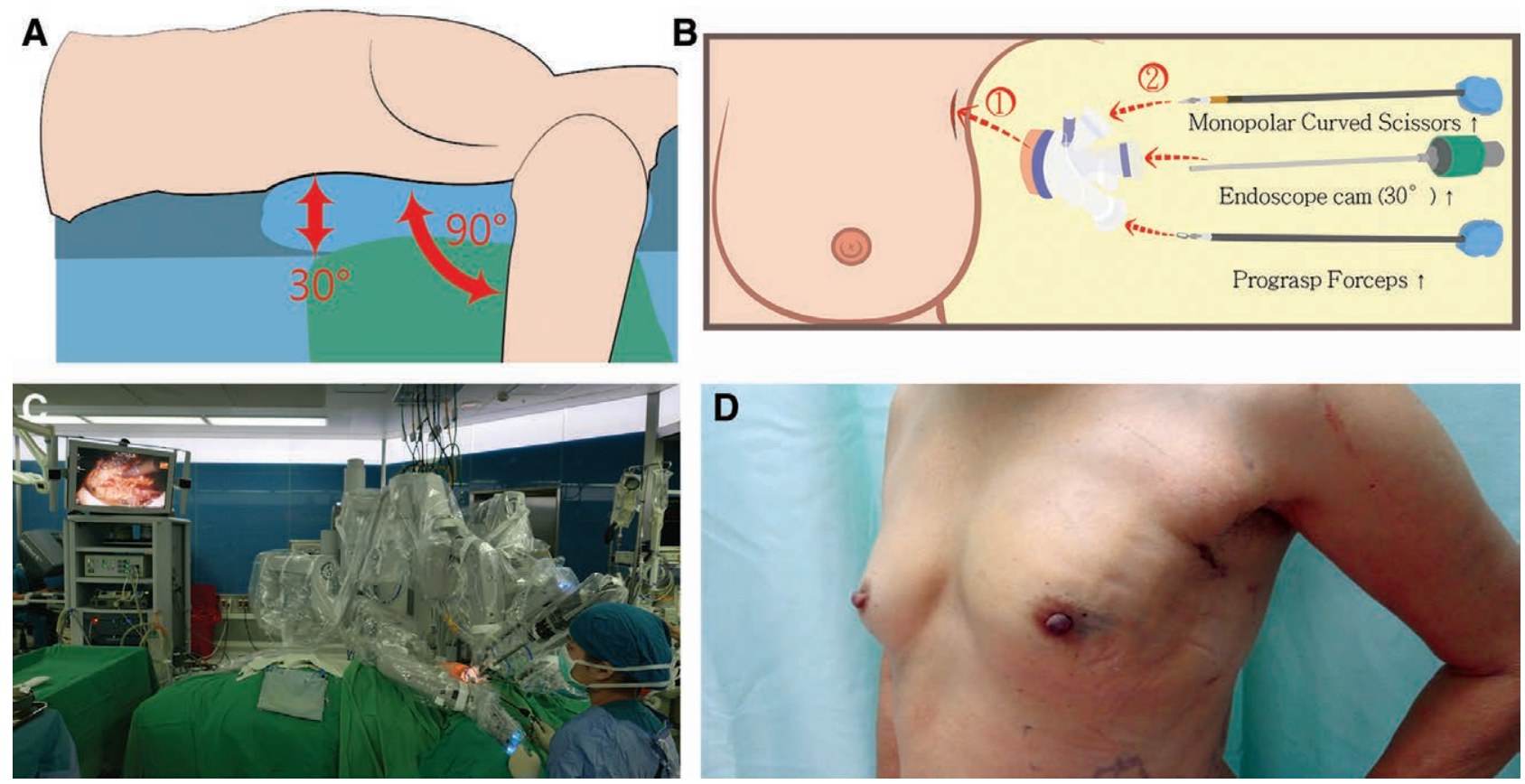

Fig. 1. Demonstration technique tips and postoperative outcome for R-NSM and IBR with Gel implant. A, The position of patients for R-NSM. The operating side shoulder was elevated to 30 degree with draping (or the arm in operating field was adducted after axillary surgery) to prevent conflict between the operating table and docking of robotic surgery system. B, Illustration of insertion of single port, and robotic surgical platform with da Vinci Si system. Video camera, monopolar scissor, and prograsp forceps. C, The setting of robotic side cart is positioned posterior to the patient with the 2 robotic arms and the endoscope extending over the patient in proximity to the ports. In this position, the arms are aligned with the plane of the breast, nearly parallel to the floor, and the ports are docked to the robotic arms. D, Postoperative lateral view showed that the wound was small and well hidden in the inconspicuous axilla region. 
tion was carried out by pulling breast tissue to create a sufficient working space. Posterior dissection was performed by detach breast tissue from pectoralis major muscle. After completion of the dissection, entire breast specimen was removed through the axillary wound. If cancer cell invasion was found in the subareolar area, the entire NAC was removed.

\section{BREAST RECONSTRUCTION}

After mastectomy, subpectoral working space was developed under direct vision by electrocautery with assistance of handle light retractor. Then single port was reinserted and gas reinflated for robotic submuscular pocket dissection. The prograsp forceps was used to lift the pectoralis major muscle, and monopolar scissor was used to cut and dissect plane. After completion of submuscular dissection with da Vinci surgical platform, the robotic instruments and single port were removed. Then, the operating table was bent into sitting position. The adequacy of submuscular pocket dissection was checked, and further dissection of submuscular pocket was performed with light source retractor. After completion of the submuscular pocket dissection, the implant was inserted from the axillary access. Usually 2 drains (beneath skin flap and submuscular pocket) were left as displayed in Video 1 (see video, Supplemental Digital Content 1, which discusses details of robotic nipple-sparing mastectomy. This video is available in the "Related Videos" section of the Full-Text article at PRSGlobalOpen.com or at http://links.lww.com/ PRSGO/A789).

\section{RESULTS}

From March to October 2017, 15 patients with breast cancer undergone R-NSM and IBR with Gel implant in our institute. The mean age of them was $46.5 \pm 10.0$ years. The pathologic stage was $30.8 \%$ DCIS, $30.8 \%$ stage I, $30.8 \%$ stage II, and $7.7 \%$ stage III. The mean operation time was $282.8 \pm 70.4$ minutes, and blood loss was $38.3 \pm 45.3 \mathrm{ml}$. The mean hospital stay was $6.7 \pm 1.2$ days. The positive surgical

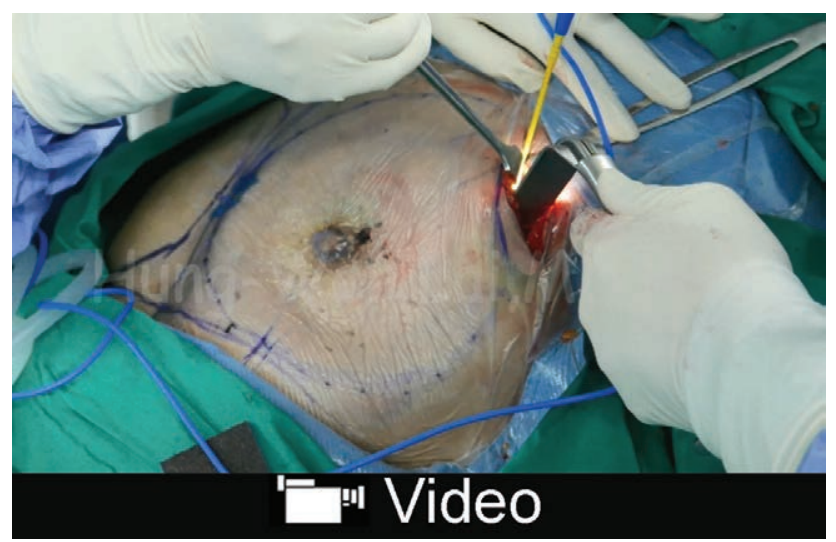

Video Graphic 1. See video, Supplemental Digital Content 1, which discusses details of R-NSM. This video is available in the "Related Videos" section of the Full-Text article at PRSGlobalOpen.com or at http://links.Iww.com/PRSGO/A789. margin rate was $0 \%$. One patient suffered from delayed axillary wound healing. Two patients $(13.3 \%)$ with transit nipple ischemia change, but no total NAC necrosis case was observed. No local recurrence, distant metastasis, or case mortality was found during mean $6.3 \pm 2.1$ months follow-up. According to the postoperative cosmetic outcome questionnaire evaluation, about $86.7 \%$ of patients graded as excellent, and $13.3 \%$ good. All patients were satisfied with the postoperative scar appearance, wound length, and wound position.

\section{DISCUSSION}

The air pressure derived from inflation of $\mathrm{CO}_{2}$ might be contributed to the reduction of bleeding, and secretion from tissue. Subcutaneous emphysema was observed as sequel after gas inflation, and usually subsided spontaneously. Robotic surgical platform with $d a$ Vinci system worked efficiently in performing subnipple biopsy. We could remove most breast glandular tissue near and beneath the NAC, and coring subnipple lactiferous ducts to minimize the risk of local recurrence. The extremity low rate of NAC and skin flap ischemia necrosis was promising. The 2 main limitations of R-NSM were the longer operation time and higher cost. ${ }^{1,5,6}$ In our initial cases, it did took longer operation time; however, after experience accumulated, the R-NSM could be decreased to within 90 minutes, and IBR with Gel implant could be completed 60 minutes after R-NSM.

From our preliminary experience, R-NSM and IBR with Gel implant is a safe procedure, with good cosmetic results, and could be a promising new technique for breast cancer patients indicated for mastectomy.

Hung-Wen Lai, $\mathbf{M D}, \boldsymbol{P h D}$
Endoscopic and Oncoplastic Breast Surgery Center
Changhua Christian Hospital
135 Nanxiao Street, Changhua 500
Taiwan
mail: 143809@cch.org.tw; hwlai650420@yahoo.com.tw

\section{ACKNOWLEDGMENTS}

The authors thank Yun-Ting Chang and Shun-Ing Tsai for the assistance in this study.

\section{REFERENCES}

1. Selber JC, Baumann DP, Holsinger FC. Robotic latissimus dorsi muscle harvest: a case series. Plast Reconstr Surg. 2012;129:13051312.

2. Dal Moro F. Robotic surgery and functional outcomes: a lesson from urology. Surg Laparosc Endosc Percutan Tech. 2014;24:392393.

3. Abramovici L, Cartier C, Pierre G, et al. Robot-assisted transaxillary thyroidectomy: surgical technique. Eur Ann Otorhinolaryngol Head Neck Dis. 2015;132:153-156.

4. Louie BE. A decade of robotics in lung cancer surgery. $J$ Thorac Dis. 2016;8:E1748-E1749.

5. Toesca A, Peradze N, Galimberti V, et al. Robotic nipple-sparing mastectomy and immediate breast reconstruction with implant: first report of surgical technique. Ann Surg. 2017;266:e28-e30.

6. Toesca A, Peradze N, Manconi A, et al. Robotic nipple-sparing mastectomy for the treatment of breast cancer: feasibility and safety study. Breast. 2017;31:51-56. 
7. Tukenmez M, Ozden BC, Agcaoglu O, et al. Videoendoscopic single-port nipple-sparing mastectomy and immediate reconstruction. J Laparoendosc Adv Surg Tech A. 2014;24:77-82.

8. Lai HW, Wu HS, Chuang KL, et al. Endoscopy-assisted total mastectomy followed by immediate pedicled transverse rectus abdominis musculocutaneous (TRAM) flap reconstruction: preliminary results of 48 patients. Surg Innov. 2015;22: 382-389.

9. Lai HW, Lin HY, Chen SL, et al. Endoscopy-assisted surgery for the management of benign breast tumors: technique, learning curve, and patient-reported outcome from preliminary 323 procedures. World J Surg Oncol. 2017;15:19.

10. Lai HW, Chen ST, Chen DR, et al. Current trends in and indications for endoscopy-assisted breast surgery for breast cancer: results from a six-year study conducted by the Taiwan Endoscopic Breast Surgery Cooperative Group. PLoS One. 2016;11:e0150310.

11. Chan SE, Liao CY, Wang TY, et al. The diagnostic utility of preoperative breast magnetic resonance imaging (MRI) and/or intraoperative sub-nipple biopsy in nipple-sparing mastectomy. Eur J Surg Oncol. 2017;43:76-84. 